\title{
A NOTE ON EXTREMA OF LINEAR COMBINATIONS OF ELEMENTARY SYMMETRIC FUNCTIONS
}

\author{
ALEXANDER KOVAČEC, SALMA KUHLMANN, AND CORDIAN RIENER
}

\begin{abstract}
This note provides a new approach to a result of Foregger 2 and related earlier results by Keilson [4] and Eberlein [1]. Using quite different techniques, we prove a more general result from which the others follow easily. Finally, we argue that the proof in [2] is flawed.
\end{abstract}

Throughout this note write $E_{j}(x)=E_{j}\left(x_{1}, \ldots, x_{n}\right), j=0,1, \ldots, n$, for the $j$-th elementary symmetric polynomial in $n$ variables. Let $H=H_{\gamma}=\left\{x \in \mathbb{R}^{n}\right.$ : $\left.E_{1}(x)=\gamma\right\}$ be a hyperplane normal to the vector $1_{n}=(1,1, \ldots, 1) \in \mathbb{R}^{n}$ and $\phi$ be a real linear combination of the $E_{j}$. Putting $D:=H \cap[0,1]^{n}$, Keilson [4] investigated the question of where the function $\phi: D \rightarrow \mathbb{R}$ assumes its extreme values. Eberlein 1] noted that the result of Keilson was actually already known by Chebyshev in 1846 and Hoeffding in 1956. He assumed $0 \leq a_{i} \leq b_{i} \leq 1$ and investigated the same question on more general domains $D^{\prime}=H \cap\left(\left[a_{1}, b_{1}\right] \times \cdots \times\left[a_{n}, b_{n}\right]\right)$. Foregger, interested in solving a problem by Pierce [7], also solved by Li [6], returned to the original Keilson-Chebyshev question, and proposed to show in [2, Theorem 2] that if $\phi$ is non-constant on $D$ (i.e. has degree at least two) and attains its extremum at an interior point of $D$, then this point must be the symmetric point of $D$, that is $\frac{\gamma}{n} 1_{n}$. At the end of his paper Foregger invites to establish similar results to his for Eberlein's domains.

In this note, we establish below in Theorem 1, Theorem 3 and Corollary 5 results from which the above mentioned are easily derived. Our method, originally developed by Riener [8] to provide an algebraic approach to Timofte's results [10] is different and much simpler: it is based on the observation that the elementary symmetric functions are closely related to roots of univariate hyperbolic polynomials, i.e. polynomials with only real roots. Given a function $f: D \subseteq \mathbb{R}^{n} \rightarrow \mathbb{R}$, write loc.extr $(f)$ for the set of local extrema of $f$; that is for the set of all points $u$ in $D$ admitting a neighborhood $N=N_{u} \subseteq D$ such that $\forall x \in N f(x) \geq f(u)$ or $\forall x \in N f(x) \leq f(u)$.

Theorem 1. If a real linear combination $\phi$ of elementary symmetric polynomials has degree at least two, then it has as a function $\phi: H \rightarrow \mathbb{R}$ on the hyperplane $H=$ $\left\{x: E_{1}(x)=\gamma\right\}$ at most one local extremum; more precisely $\operatorname{loc} . \operatorname{extr}(\phi) \subseteq\left\{\frac{\gamma}{n} 1_{n}\right\}$.

We will need an auxiliary lemma.

Lemma 1. Let $f \in \mathbb{R}[t]$ be hyperbolic of degree $n$ with only $s \in\{1, \ldots, n-1\}$ distinct (real) roots all lying in some open interval $I$. Then there exists $g \in \mathbb{R}[t]$ of degree

Date: February 23, 2011.

1991 Mathematics Subject Classification. Primary 13J30; Secondary 26D05.

Key words and phrases. Extrema, elementary symmetric functions, hyperbolic polynomials. 
$n-s$ so that for all small $\varepsilon>0$, the polynomials $f+\varepsilon g$ and $f-\varepsilon g$ are hyperbolic and have both more than $s$ distinct roots all lying in $I$.

Proof. Let $x_{1}, \ldots, x_{s}$, be the distinct zeros of $f$ which by hypothesis all lie in $I$. Without loss of generality assume $I$ bounded and for notational convenience assume $I=] x_{0}, x_{s+1}\left[\right.$ and $x_{0}<x_{1}<\ldots<x_{s}<x_{s+1}$. We have a factorization

$$
f=\prod_{i=1}^{s}\left(t-x_{i}\right) \cdot g_{1}=: p \cdot g_{1}
$$

where the roots of $g_{1}$ are all in $\left\{x_{1}, \ldots, x_{s}\right\}$ and $g_{1}$ is of degree $n-s \geq 1$. Now for $x_{i}<t<x_{i+1}, i=0, \ldots, s, \operatorname{sgn} p(t)=(-1)^{s-i}$. Therefore there exist points $\left.\xi_{i} \in\right] x_{i}, x_{i+1}$ [, and $\varepsilon>0$, such that if $s-i$ is odd, $p\left(\xi_{i}\right)<-2 \varepsilon$, while if $s-i$ is even, $p\left(\xi_{i}\right)>2 \varepsilon$. Therefore the polynomials $p \pm \varepsilon$ have in $\xi_{0}, \xi_{1}, \xi_{2}, \ldots, \xi_{s-1}, \xi_{s}$ alternating signs. So they have in each of the intervals $] \xi_{i}, \xi_{i+1}[, i=0,1, \ldots, s-1$, a root. Therefore $p \pm \varepsilon$ both have $s$ distinct real roots and so are hyperbolic and the roots all lie in $I$. Furthermore we see that $p \pm \varepsilon$ have none of their roots in the set $\left\{x_{1}, \ldots, x_{s}\right\}$. Hence $(p \pm \varepsilon) \cdot g_{1}=f \pm \varepsilon g_{1}$ are hyperbolic and each have more than $s$ distinct roots all lying in $I$.

Proof of Theorem 1. If the set of local extrema of $\phi: H \rightarrow \mathbb{R}$ is nonempty, choose a local extremum, say $a:=\left(a_{1}, \cdots, a_{n}\right)$, so that the number $s:=\left|\left\{a_{1}, \ldots, a_{n}\right\}\right|$ of distinct components of $a$ is maximal and consider the univariate polynomial

$$
f_{a}:=\prod_{i=1}^{n}\left(-a_{i}+t\right)=\sum_{i=0}^{n-2}(-1)^{n-i} E_{n-i}(a) t^{i}-\gamma t^{n-1}+t^{n} .
$$

Here we used Viète's formula and to facilitate reading of the following we think of $f_{a}$ and similar univariate polynomials as written from left to right with rising powers and write $\phi$ as $\phi(x)=\sum_{i=0}^{n-2} c_{n-i} E_{n-i}(x)+c_{1} E_{1}(x)+c_{0} E_{0}(x)$. Assume $s \in\{2,3, \ldots, n-1\}$. By lemma 2 there exists a polynomial $g=\sum_{i=0}^{n-s} g_{n-i} t^{i}$ of degree $n-s$, and an $\varepsilon_{0}>0$ such that for all $\varepsilon$ with $0<|\varepsilon|<\varepsilon_{0}$, the polynomial $f_{a}+\varepsilon g$ is hyperbolic and has at least $s+1$ distinct roots. We can represent for every $\varepsilon$ the $n$ (real) roots of $f_{a}+\varepsilon g$ as the entries of $a(\varepsilon)=\left(a_{1}(\varepsilon), \ldots, a_{n}(\varepsilon)\right.$ ), and according to Kato [3, p.109] think of $a($.$) actually as a continuous function. We$ then have a similar equation as above for $f_{a}+\varepsilon g$ (in place of $f_{a}$ ) and $E_{n-i}(a(\varepsilon)$ ) (in place of $\left.E_{n-i}(a)\right)$. We find $(-1)^{n-i} E_{n-i}(a(\varepsilon))=(-1)^{n-i} E_{n-i}(a)+\varepsilon g_{n-i}$, where for $i>n-s$, we put $g_{n-i}=0$. In particular $E_{1}(a(\varepsilon))=\gamma$. By these formulae we find

$$
\phi(a(\varepsilon))=\phi(a)+\varepsilon \sum_{i=0}^{n-s}(-1)^{n-i} c_{n-i} g_{n-i} .
$$

It now follows for all $\varepsilon$ of small modulus and appropriate sign, that $\phi(a(-\varepsilon)) \leq$ $\phi(a) \leq \phi(a(\varepsilon))$ with $a( \pm \varepsilon) \in O_{a} \subseteq H, O_{a}$ a neighborhood of $a$. So in every neighborhood of $a$ there are points $a(-\varepsilon)$ at which $\phi$ is not larger than $\phi(a)$ and $a(\varepsilon)$ at which $\phi$ is not smaller than $\phi(a)$ and which have at least $s+1$ distinct coordinates. This contradicts our choice of $a$. Therefore $s \in\{1, n\}$, that is, $f_{a}$ has either one root of multiplicity $n$, or $n$ distinct roots. Assume $s=n$. Since degree $(\phi) \geq 2$, $\left(c_{2}, \ldots, c_{n}\right) \neq 0$. Thus there exist reals $g_{2}, \ldots, g_{n}$ so that $\sum_{i=0}^{n-2} c_{n-i} g_{n-i} \neq 0$. Consider the polynomial $g=\sum_{i=0}^{n-2} g_{n-i} t^{i}$ and note that $f_{a}+\varepsilon g$ will have for all $\varepsilon$ of 
small modulus $n$ roots. With the reasoning above, we infer this time strict inequalities $\phi(a(-\varepsilon))<\phi(a)<\phi(a(\varepsilon))$, again arriving at a contradiction. Hence $s=1$ and $a=\frac{\gamma}{n} 1_{n}$, as we wished to show.

[2, Theorem 2] follows at once from Theorem [1 If $\phi$ is non constant on $D$ then it is nonconstant on the hyperplane $H$ of our theorem, hence evidently must have degree at least two (that is some of the polynomials $E_{2}, \ldots, E_{n}$ must occur in $\phi$ ). If $\phi$ attains its maximum or minimum at an interior point $P$ of $D$ then a small enough neighbourhood of $P$ as a point of $D$ is also a neighbourhood of $P$ as a point in $H$. So $P$ is a local extremum as well for the function $\phi: D \rightarrow R$ as for its extension $\phi: H \rightarrow R$. Hence by our theorem $P$ must be the symmetric point as claimed by Foregger.

A simple adaptation of the proof of Theorem 1, which we leave to the reader, yields the following generalization of Theorem 1 which will not be further used in this paper.

Theorem 2. If the polynomial $\phi$ of theorem 1 has degree at least $k+1$ and is considered as a function on the real variety $\left\{x: E_{1}(x)=\gamma_{1}, \ldots, E_{k}(x)=\gamma_{k}\right\}$, then each of its local extrema $a$ has at most $k$ distinct components, i.e. $\left|\left\{a_{1}, \ldots, a_{n}\right\}\right| \leq k$.

We can now derive a corollary in the spirit of Eberlein and Foregger which is more complete. The following notation is fixed through the rest of the paper. Let $a_{i}<b_{i}$, $i=1, \ldots, n$ be real numbers. Denote by $e_{i}=(0, \ldots, 0,1,0, \ldots 0)$, the $i$-th standard vector in $\mathbb{R}^{n}$. Using the $(2 n+2) \times n$ matrix

$$
A=\left[-e_{1}^{\top}, e_{1}^{\top},-e_{2}^{\top}, e_{2}^{\top}, \ldots,-e_{n}^{\top}, e_{n}^{\top},-1_{n}^{\top}, 1_{n}^{\top}\right]^{\top}
$$

(whose rows are $-e_{1}, e_{1},-e_{2}, \ldots$ ) and the column

$$
d=\left(-a_{1}, b_{1},-a_{2}, b_{2}, \ldots,-a_{n}, b_{n},-\gamma, \gamma\right)^{\top},
$$

the set $D^{\prime}=H \cap\left(\left[a_{1}, b_{1}\right] \times \cdots \times\left[a_{n}, b_{n}\right]\right)$ can be defined as $D^{\prime}=\{x: A x \leq d\}$. Since $D^{\prime}$ is bounded it is a polytope in the sense of Schrijver [9, p. 89]. For $K \subseteq\{1, \ldots, n\}$ we write $K^{c}=\{1, \ldots, n\} \backslash K$ for its complement. We begin by characterizing the faces of $D^{\prime}$.

Lemma 2. A subset $F$ of $\mathbb{R}^{n}$ is face of $D^{\prime}$ if and only if it is nonempty and there exists a subset $K$ in $\{1,2, \ldots, n\}$ and $u_{l} \in\left\{a_{l}, b_{l}\right\}$ for all $l \in K^{c}$, so that

$$
F=\left\{\sum_{k \in K} x_{k} e_{k}+\sum_{l \in K^{c}} u_{l} e_{l}: \forall k \in K a_{k} \leq x_{k} \leq b_{k} \text { and } \sum_{k \in K} x_{k}+\sum_{k \in K^{c}} u_{k}=\gamma\right\} .
$$

If $K^{c}$ is a maximal set such that the inequality conditions at the right of the ':' are simultaneously satisfiable without equalities, then the affine dimension of $F$ is $(|K|-1)^{+}$.

Proof. By [9, p101], a set $F \subseteq \mathbb{R}^{n}$ is a face of $D^{\prime}$ iff $F \neq \emptyset$ and $F=\{x: A x \leq$ $d$ and $\left.A^{\prime} x=d^{\prime}\right\}$, where $A^{\prime}$ and $d^{\prime}$ are obtained from $A, d$, respectively, by selecting the same row indices. It may happen that there exists a further, say $i$-th row $a_{i, *}$ of $A$, such that $a_{i, *} x=d_{i}$ for all $x \in F$. We think from now on of all such rows as included in $A^{\prime}$ and write $A^{=}$for this submatrix of $A$, and $d^{=}$for the corresponding subvector of $d$. Let $A^{+}$be the complementary submatrix of $A^{=}$in $A$. Then for each row index $i$ of $A$ defining a row of $A^{+}$there exists a vector $x^{(i)} \in F$ such that $A^{+} x^{(i)}<d_{i}$. The arithmetic mean of all such vectors yields a vector $x \in F$ so that $A^{+} x<d^{+}$(meaning that all component inequalities are strict). We note that $F$ can be written as $F=\left\{x: A^{+} x \leq d\right.$ and $\left.A^{=} x=d^{=}\right\}$. The set of row indices of $A$ defining $A^{=}$contains evidently $\{2 n+1,2 n+2\}$ but contains from each of 
the sets $\{2 i-1,2 i\}, i=1, \ldots, n$ at most one index, since otherwise $F$ would be empty. An index lying in $\{2 i-1,2 i\}$ fixes $x_{i}$ to be equal to some $u_{i} \in\left\{a_{i}, b_{i}\right\}$. Let $K^{c} \subseteq\{1, \ldots, n\}$ be the set of indices $i$ of variables so obtained, viewed as the complement of some set $K \subseteq\{1, \ldots, n\}$. Then the components $x_{k}, k \in K$ of $x \in F$ may satisfy $a_{k}<x_{k}<b_{k}$ and $\sum_{k \in K} x_{k}=\gamma-\sum_{k \in K^{c}} u_{k}$. It is now clear that $F$ can be written as above and the affine dimension of $F$ (which is by definition the dimension of its affine hull) equals $(|K|-1)^{+}$.

Corollary 3. Assume that as a function, $\phi: D^{\prime} \rightarrow \mathbb{R}$ is nonconstant on every face of dimension one (edge) of the polytope $D^{\prime}$. Then every local extremum $p$ of $\phi$ is point in a cartesian product $\left\{a_{1}, e, b_{1}\right\} \times \cdots \times\left\{a_{n}, e, b_{n}\right\}$. Here $e$ has to be chosen such that the sum of the components of $p$ is $\gamma$.

Proof. Let $p \in D^{\prime}$ be a local extremum of $\phi$. Since the faces of $D^{\prime}$ form a lattice [9, section 8.6], there exists a unique face $F$ of minimal dimension containing $p$. Then $p \in \operatorname{int} F$. We think of $F$ as written in the above lemma, with the set $K^{c}$ there chosen maximal. If $\operatorname{dim} F=0$, then $p$ is a vertex, $F=\{p\}$, and $|K|=0$ or $|K|=1$. It then follows by the above characterization of $F$ that $p=\left(p_{1}, \ldots, p_{n}\right)$ is a point so that for at least $n-1$ indices $i, p_{i} \in\left\{a_{i}, b_{i}\right\}$. Consequently $p$ lies in one of the cartesian products admitted. Assume now $\operatorname{dim} F=$ $k \geq 1$. Then $F$ has a nonempty relative interior given via $\gamma^{*}=\gamma-\sum_{k \in K^{c}} u_{k}$ by $\operatorname{int} F=\left\{\sum_{k \in K} x_{k} e_{k}+\sum_{l \in K^{c}} u_{l} e_{l}: \forall k \in K a_{k}<x_{k}<b_{k}\right.$ and $\left.\sum_{k \in K} x_{k}=\gamma^{*}\right\}$.

Now $F$ has faces of $D^{\prime}$ which have dimension 1 [9, section 8.3] on which by assumption $\phi$ is not constant; consequently $\phi$ is not constant on the affine hull of $F$, and hence is a polynomial of degree $\geq 2$ on it. The local extremum $p \in \operatorname{int} F$ of $\phi$ is necessarily also local extremum of the restriction of $\phi$ to the affine hull of $F$. Now this restriction is $\phi^{\prime}\left(x_{k_{1}}, \ldots, x_{k_{|K|}}\right)=\phi^{\prime}\left(\sum_{k \in K} x_{k} e_{k}\right):=\phi\left(\sum_{k \in K} x_{k} e_{k}+\sum_{l \in K^{c}} u_{l} e_{l}\right)$. It is again a certain real linear combination of elementary symmetric functions of variables $x_{k}, k \in K=\left\{k_{1}, \ldots, k_{|K|}\right\}$ : this follows since a similar fact holds for each of the elementary symmetric functions. Applying our main result to the affine hull of $F$, we get that $p=\sum_{k \in K} \frac{\gamma^{*}}{|K|} e_{k}+\sum_{l \in K^{c}} u_{l} e_{l}$, as we wished to show.

Remark 4. a. The mentioned authors state some of their results in somewhat unprecise terms. Eberlein [1, Theorem 1 p. 312] tells us that the 'minimum and the maximum of $\phi$ is assumed at least among the points whose components which are not endpoints are all equal. Moreover if the maximum and the minimum is attained only in the interior of $D^{\prime}$ then it is assumed uniquely at the point $\frac{\gamma}{n} 1_{n}$. After a more precise formulation, the corresponding proof is right but unfortunately does not give more. In particular it does not exclude for nonconstant $\phi$ lines on which $\phi$ has constant extreme value and which extend to the boundary of $D^{\prime}$. Thus again there may be interior extrema other than $\frac{\gamma}{n} 1_{n}$. This possibility is what Foregger proposed to exclude for his domain $D$. He attempts establishing his result by induction over dimension via reasoning not easily adaptable to Eberlein's more general domain $D^{\prime}$. We now discuss the error in Foregger's proof, and point out another mistake in his paper.

b. (i) Using exactly his notation, in [2, p.384], Foregger derives for $s<n$, $c \in \mathbb{R}^{n-s}$ constant, and $y \in \mathbb{R}^{s}$, the first two lines in the following chain; the third line is a consequence of noting that $E_{0}(y)=1$, and $E_{1}(y)=\gamma^{*}$ by Foregger's (local) 
definition (of) $C_{\gamma^{*}}=\left\{y \in \mathbb{R}^{s}: \sum_{i=1}^{s} y_{i}=\gamma^{*}, y_{i} \in[0,1]\right\} \subset \mathbb{R}^{s}$ on p. 383:

$$
\begin{aligned}
0 & =\phi^{*}(y) \\
& =\sum_{k=0}^{n} E_{k}(y) \sum_{r=k}^{n} c_{r} E_{r-k}(c)-\sum_{r=2}^{n} c_{r} E_{r}(0, c) \\
& =\sum_{r=0}^{n} c_{r} E_{r}(c)+\gamma^{*} \sum_{r=1}^{n} c_{r} E_{r-1}(c)-\sum_{r=2}^{n} c_{r} E_{r}(0, c)+\sum_{k=2}^{n} E_{k}(y) \sum_{r=k}^{n} c_{r} E_{r-k}(c) .
\end{aligned}
$$

Note that $y \in \mathbb{R}^{s}$, so the definition of the elementary symmetric functions requires $E_{s+1}(y)=\cdots=E_{n}(y)=0$, a fact not observed in [2]. Granted that as functions $1, E_{2}, E_{3}, \ldots, E_{s}$ are (usually) linearly independent on $C_{\gamma^{*}}$ - to see this put $n$ 1 variables equal to real variable $t$, the other equal to $\gamma^{*}-t$ and observe that $E_{j}\left(t, \ldots, t, \gamma^{*}-t\right)$ is a polynomial of degree $j$ in $t$ - we may infer the equations $\sum_{r=k}^{n} c_{r} E_{r-k}(c)=0, k=2,3, \ldots, s$. The problem in [2] is that these equations are claimed for $k=2, \ldots, n$, (and not only for $k=2, \ldots, s$ ) and then used in the order $k=n, n-1, \ldots, 1$, to derive that $c_{n}, c_{n-2}, \ldots, c_{1}$, are 0 . Therefore the proof seems to be beyond repair.

(ii) Foregger in examining Eberlein's theorem, claims on p. 385 that the function $\phi=\phi(x, y, z)=x y z-0.5(x y+x z+y z)$ assumes on $C_{5 / 4}=\{(x, y, z): x+y+z=$ $5 / 4\} \cap([3 / 8,5 / 8] \times[3 / 8,5 / 8] \times[1 / 8,3 / 8])$ in $p_{0}=(1 / 2,1 / 2,1 / 4)$ an interior maximum of value $-0.1875=\phi\left(p_{0}\right)$. It is easily seen that this would contradict his own (and our) main theorem. Indeed numerical experiments indicate that $p_{0}$ is not an interior local extremum.

c. In 11, Waterhouse gives examples showing that the symmetric point in general needs not be a local extremum of symmetric functions subject to symmetric conditions. We thank the referee to have pointed out that $\phi(x)=E_{2}(x)-E_{3}(x)$ is an example of a function that satisfies the hypothesis of our Theorem 1 with $n=\gamma=3$ but has no extremum at all. Indeed $\mathbb{R} \ni t \mapsto p(t)=(1+t, 1+t, 1-2 t)$ describes a line in $H$ through the point $p(0)=(1,1,1)$ and $\phi(p(t))=2+2 t^{3}$. Examples for larger $n$ can be similarly constructed.

Acknowledgment: We thank António Leal Duarte for having drawn our attention to Kato's book. 


\section{REFERENCES}

[1] P. J. Eberlein, Remarks on the van der Waerden conjecture, II, Linear Algebra and its Applications 2: 311-320 (1969).

[2] T. H. Foregger, On the Relative Extrema of a Linear Combination of Elementary Symmetric Functions, Linear and Multilinear Algebra 20: 377-385 (1987).

[3] T. Kato, Perturbation Theory of Linear Operators, Springer 1966.

[4] J. Keilson, A theorem on optimum allocation for a class of symmetric multilinear return functions, J. Math. Anal. and Appl. 15: 269-272 (1966).

[5] J. Keilson, On global extrema for a class of symmetric functions, J. Math. Anal. and Appl. 18: 218-228 (1967).

[6] C. K. Li, An inequality on Elementary Symmetric Functions, Linear and Multilinear Algebra 20: 373-375 (1987).

[7] S. Pierce, Research problem: Elementary symmetric Functions, Linear and Multilinear Algebra 17: p. 375 (1985).

[8] C. Riener, On the degree and half degree principle for symmetric polynomials, to appear in Journal of Pure and Applied Algebra, arXiv:1001.4464v1.

[9] A. Schrijver, Theory of Linear and Integer Programming, J. Wiley, 1986.

[10] V. Timofte, On the positivity of symmetric polynomial functions. Part I: General results, $J$. Math. Anal. Appl. 284, 174-190 (2003).

[11] W. C. Waterhouse, Do symmetric problems have symmetric solutions?, Amer. Math. Monthly 90: 378-387 (1983).

Departamento de Matemática, Faculdade de Ciências e Tecnologia, Apartado 3008, EC Universidade, P 3001-454 Coimbra, Portugal

E-mail address: kovacec@mat.uc.pt

Fachbereich Mathematik und Statistik, Universität Konstanz, 78457 Konstanz, GerMANY

E-mail address: salma.kuhlmann@uni-konstanz.de

Institut für Mathematik, Goethe Universität, 60325 Frankfurt, Germany

E-mail address: riener@math.uni-frankfurt.de 